\title{
An Ionic Polymer Bead-Supported Lipid System Using Naturally Occurring Phospholipids
}

\author{
Samuel Osei-Asante, Mamoru Haratake*, Takeshi Fuchigami \\ and Morio Nakayama* \\ Graduate School of Biomedical Sciences, Nagasaki University \\ Nagasaki, Japan 852-8521
}

\begin{abstract}
*Authors to whom correspondence should be addressed.
E-mail: haratake@nagasaki-u.ac.jp; morio@nagasaki-u.ac.jp
\end{abstract}

Figures 3 and 4 appear in color online: http://jbc.sagepub.com 
ABSTRACT: Spherically supported bilayer membranes on cationic polymer beads were prepared from naturally occurring phospholipids [phosphatidylcholine (PC) and phosphatidylserine (PS)]. The amount of phospholipids bound to the cationic polymer beads from the liposomal suspensions of various lipid compositions increased with increases in anionic PS. Confocal fluorescence microscopic observations with a fluorescence membrane probe indicated that the phospholipid molecules in the phospholipid-bead complexes were along the surface of the beads. Negatively charged trypan blue dye was preferably bound to the phospholipid-free beads by electrostatic attractive interaction, but was excluded from the phospholipidbead complexes due to the bilayer membrane structure formed on the beads. This method could provide a better mimetic of biological membranes in comparison to physically unstable conventional liposomes.

KEY WORDS: bilayer membrane, confocal fluorescence microscopy, phospholipid, polymer bead 


\section{INTRODUCTION}

Liposomes are artificial closed-vesicles of lipid bilayer membranes. Since the first description of liposomes by Bangham in 1965 [1], they have been widely used as simplified models to obtain basic information on the structure-function relationship of biomembranes in the fields of chemical and/or physical biology [2-4]. Most liposomes have much smaller diameters (submicrometers) and higher curvature than the biological cells, although, liposomes with diameters up to $100 \mu \mathrm{m}$ have been developed [5-7]. The yield of liposomes with diameters greater than $10 \mu \mathrm{m}$ is often lower under high-salt conditions [8]. The poor mechanical stability is due to their hollow structure which also impedes their use as large-sized liposomes, (giant liposomes), as model biological membranes.

An effective means to overcome the drawbacks of large-sized liposomes is to use supporting materials under the lipid bilayer membranes. Lipid bilayers on solid supports were developed two decades ago as a model system for biological membranes [9]. It was found that he lateral and rotational mobility of lipid molecules in the bilayer membranes was preserved, as the lipid bilayer was not covalently coupled with supporting materials: a thin layer of water between the solid supports and the lipid bilayers $(\sim 1-2 \mathrm{~nm})$ maintained the dynamic properties of free lipid bilayers. Spherically supported membranes are thought to assemble around micron sized beads, such as silica and glass beads, by essentially a vesicle fusion process [5,10-13]. Such systems provide membrane morphologies that can be easily controlled with good reproducibility and stability $[14,15]$. Recently, a more sophisticated system, in which lipid bilayers are tethered to the support through the avidin-biotin binding, was reported [16]. Despite the amount of research on vesicle 
adsorption during the last two decades [17-19], there is still no clear picture of the forces and various events that a vesicle goes through as it adheres, fuses and spreads on a solid surface.

Consequently, we developed a novel methodology for the preparation of a polymer bead-supported lipid system, in which the electrostatic attractive interactions between lipids and polymer beads serve as "molecular glue" to immobilize lipid bilayer membranes on polymer beads [20]. When a cationic polymer bead-supported synthetic anionic lipid bilayer membrane system was prepared from naturally occurring phospholipids and applied to a packing material for ion-exchange chromatography. The ionic property of the lipid bilayer surface was reflected in the retention behavior of selected proteins [21]. In this study, the ionic polymer beadsupported lipid systems were prepared from naturally occurring phospholipids. 


\section{EXPERIMENTAL}

\section{Materials}

Dioleoylphosphatidylserine and egg yolk phosphatidylcholine (hydrogenated) were purchased from NOF Corporation (Tokyo, Japan). DIAION SA11A (a styrenedivinylbenzene-based non-porous quaternary ammonium type anion-exchange bead with a $0.35-0.55 \mathrm{~mm}$ in diameter and its ammonium nitrogen content is 0.85 $\mathrm{mmol} / \mathrm{mL}$ polymer in water) was obtained from Mitsubishi Chemical Co. (Tokyo, Japan). Octadecylrhodamine B (ODR) chloride was from Molecular Probes, Inc. (Eugene, OR, U.S.A.). Trypan blue dye was obtained from Nacalai Tesque Inc. (Kyoto, Japan). Water used in this study was generated using a Milli-Q Biocel system. All other chemicals were of commercial reagent grade and used as received.

\section{Preparation of Phospholipid-SA11A Complex}

Phospholipids $(25 \mu \mathrm{mol})$ were placed in a round-bottom flask and dissolved by adding $5 \mathrm{~mL}$ of chloroform. The chloroform was gently evaporated under reduced pressure. After the addition of $50 \mathrm{~mL}$ of $0.05 \mathrm{M}$ Tris-HCl buffer $(\mathrm{pH} 8.5)$ to the flask, the mixture was vortexed for $1 \mathrm{~h}$. The milky suspension obtained was sonicated using a probe (Sonicator 250D, Branson, Danbury, CT) at $80 \mathrm{~W}$ for $15 \mathrm{~min}$. Ten milliliters of the liposomal suspension were shaken with $1 \mathrm{~mL}$ of SA11A at $55^{\circ} \mathrm{C}$ for $30 \mathrm{~min}$. The obtained phospholipid-bead complexes were thoroughly washed with $0.05 \mathrm{M}$ Tris- $\mathrm{HCl}$ buffer $(\mathrm{pH} \mathrm{8.5)}$ and kept in the washing buffer at room temperature until use. 


\section{Zeta Potential and Mean Particle Diameter of the Liposomes}

Zeta potential and mean particle diameter of the liposomes used for the preparation of the phospholipid-SA11A complexes were determined using an ELS7500 (Otsuka Electronics Co., Ltd., Osaka, Japan) and a Zetasizer Nano ZS (Malvern Instruments Ltd., Worcestershire, UK), respectively.

\section{Determination of the Phosphorous Content of the Complex}

After wet digestion of the lipid vesicle suspensions, using a 4:1 mixture of nitric acid and perchloric acid, the phosphorous content was determined based on vanadium $(\mathrm{V})$ which is capable of forming a yellow complex with phosphate ion [22]. The color produced by the complexation was monitored at $440 \mathrm{~nm}$. Phosphorous amount of the beads was calculated from the difference in phosphorous concentration of the liposomal suspension between before and after preparation of the phospholipid-SA11A complexes. Phosphorous amount in SA11A beads used was less than $0.01 \mu \mathrm{g} / \mathrm{mL}-\mathrm{SA} 11 \mathrm{~A}$.

\section{Octadecylrhodamine B Labeling and Fluorescence Microscopic Observation}

Half milliliter of ODR ethanolic solution $(25 \mu \mathrm{g} / \mathrm{mL})$ was added to the $50 \mathrm{~mL}$ of liposomal suspensions just before sonication, as reported by Sunamoto et al. [23]; the phosphollipid-SA11A complexes with ODR were prepared as described above. Confocal laser scanning fluorescence microscopic observations were performed on an LSM 710 equipped with operation software ZEN 2008 (Carl Zeiss, Inc., Tokyo, 
Japan). An objective with a 10 -fold magnification and a 1.15 numerical aperture was used to detect fluorescence emission excited by a He-Ne $543 \mathrm{~nm}$ laser beam (1 mW).

\section{Trypan Blue Exclusion Test}

Phospholipid-SA11A complexes or lipid-free SA11A was put into $0.002 \mathrm{w} / \mathrm{v} \%$ trypan blue solution, and allowed to stand for $1 \mathrm{~h}$ at room temperature with gentle shaking occasionally. After washed thoroughly with $0.05 \mathrm{M}$ Tris-HCl buffer $(\mathrm{pH} 8.5)$, the specimens in wet state were observed under a light microscope SMZ-800 (Nikon, Tokyo, Japan). 


\section{RESULTS AND DISCUSSION}

The phospholipids-cationic bead complexes were prepared by a simple method; the liposomal suspensions from phosphatidylcholine (PC) and phosphatidylserine (PS) were mixed with the beads at temperature of which PC and PS are in lamella states. In our methodology, the adhesion of the liposomes onto the surface of the beads by electrostatic interactions and subsequent liposomal membrane fusion and restructuring to the continuous phospholipid bilayer membrane structure was expected. The mean particle diameters and zeta potential of liposomes used for the preparation of the phospholipid-SA11A complexes are summarized in Table 1 and Figure 1, respectively. PS-containing liposomes were colloidal particles ranging from 40 to $100 \mathrm{~nm}$. The magnitude of zeta potential, a measure of the mobility of colloidal particles in electric field, increased with increases of anionic PS in the liposomes. With increasing PS in the liposomes, the amount of phosphorous in the phospholipids-SA11A complexes obtained also increased (Figure 2). This indicated that electrostatic attractive interaction between anionic PS and cationic SA11A plays a role during the formation of the phospholipids-SA11A complexes.

To examine the location of the liposomal membranes in the phospholipids-SA11A complexes, a fluorescence membrane probe [octadecylrhodamine B (ODR)] was added to the liposomes during the preparation of the complexes. The ODR-labeled complexes were subjected to confocal laser scanning fluorescence microscopy to check the cross sectional views. For all of the phospholipids-SA11A complexes, ODR fluorescence was only observed along the outline of the beads and not inside the beads (Figure 3). These images clearly indicate that the phospholipid bilayer membranes formed are localized on the outer surface of SA11A beads. When the 
complexes were prepared by the reverse phase evaporation method [20] using PC and PS mixtures of similar composites, ODR fluorescence was also found in the same location of the complexes with no fluorescence in the inner part of the beads (data not shown).

The membrane integrity on the surface of the complexes was tested using the Trypan blue (TB)-exclusion method which is commonly used for counting the number of viable cells in cell culture experiments [24]. TB leaks into cells that have grossly disrupted plasma membranes, and hence its uptake denotes whether cells are viable or not. The sulfonic groups, on the TB molecule, bind strongly to SA11A beads by electrostatic attractive force with ammonium groups on SA11A. Although the phospholipid assemblies locate on the outer surface of SA11A, the liposomes and/or their aggregates may simply adhere onto the surface of the beads without any structural changes of the liposomes, such as membrane fusion. If so, an anionic lowmolecular weight species, such as TB (Mw 960.8) can easily pass through the liposomes or their aggregates, and bind to cationic SA11A beads. If SA11A are fully covered with continuous phospholipid bilayer membrane structures without any defects or edges, the complexes can exclude TB molecule, as do living cells. When both the phospholipid-free SA11A and the complexes were soaked in a TB solution, the SA11A was stained blue, while no staining was observed for the phospholipidSA11A complexes (Figure 4). Therefore, SA11A was thought to be covered by phospholipid-closed membranes well enough to exclude TB molecule. In PC, PSSA11A complexes, PS molecule would preferentially locate in the inner leaflet in the membranes due to its anionic character rather than neutral PC molecule. Similarly, PS also normally localize to the inner leaflet of the plasma membranes of living cells. 
Therefore, our method could provide a better mimetic of an actual plasma membrane in comparison to conventional liposomes and other solid material support systems.

In conclusion, bilayer membranes involving negatively charged phospholipids were successfully immobilized on the surface of positively charged polymer beads on the basis of the electrostatic interaction between the phospholipids and the beads. The polymer bead acted as a scaffold material, just like the cytoskeletal filaments, that reinforce the physical structure of the plasma membranes of biological cells [25]. This preparation technique may be applicable for the immobilization of native membranes derived from living cells on spherical supports and hence provide a means to reconstitute membrane proteins on solid support for the study of membrane proteins. We are now examining the reconstitution of the plasma membranes of red blood cells on the cationic SA11A beads. 


\section{REFERENCES}

1. Banghama, A.D., Standishl, M.M. and Weissmann, G. (1965). The Action of Steroids and

Streptolysin S on the Permeability of Phospholipid Structures to Cations, J. Mol. Biol., 13: 253-259.

2. Evans, E.A. (1974). Bending Resistance and Chemically Induced Moments in Membrane Bilayers, Biophys. J., 14: 923-931.

3. Lipowsky, R. (1991). The Conformation of Membranes, Nature, 349: 475-481.

4. Sackmann, E. (1996). Supported Membranes: Scientific and Practical Applications, Science, 271: 43-48.

5. Miyata, H. and Hotani, H. (1992). The Morphological Changes in Liposomes Caused by Polymerization of Encapsulated Actin Bundles, Proc. Nat. Acad. Sci. U. S. A., 89: 1154711551.

6. Mathivest, L., Cribier, S. and Devaux, P.F. (1996). Shape Change and Physical Properties of Giant Phospholipid Vesicles Prepared in the Presence of an AC Electric Field, Biophys. J., 70: 1112-1121.

7. Mengaer, F.M. and Keiper, J.S. (1998). Chemistry and Physics of Giant Vesicles as Membrane Models, Curr. Opin. Chem. Biol., 2: 726-732.

8. Yamashita, Y., Oka, M., Tanaka, T. and Yamazaki, M. (2002). A New Method for the Preparation of Giant Liposomes in High Salt Concentrations and Growth of Protein Microcrystals in Them, Biochem. Biophys. Acta, 1561: 129-134.

9. Tamm, L.K. and McConnell, H.M. (1985). Supported Phospholipid Bilayers, Biophys. J., 47: 105-113.

10. Bayeri, T.M. and Bloom M. (1990). Physical Properties of Single Phospholipid Bilayers Adsorbed to Micro Glass Beads, Biophys. J., 58: 357-362.

11. Korlach, J., Schwille, P., Webb, W.W. and Feigenson, G.W. (1999). Characterization of Lipid Bilayer Phases by Confocal Microscopy and Fluorescence Correlation 
Spectroscopy, Proc. Nat. Acad. Sci. U. S. A., 96: 8461-8466.

12. Buranda, T., Huang, J, Ramarao, G.V., Ista, L.K., Larson, R.S., Ward, T.L., Sklar, L.A. and Lopez, G.P. (2003). Biomimetic Molecular Assemblies on Glass and Mesoporous Silica Microbeads for Biotechnology, Langmuir, 19: 1654-1663.

13. Baksh, M.M., Jaros, M. and Groves, J.T. (2004). Detection of Molecular Interactions at Membrane Surfaces through Colloid Phase Transitions, Nature, 427: 139-141.

14. Troutier, A.-L. and Ladavière, C. (2007). An Overview of Lipid Membrane Supported by Colloidal Particles, Adv. Colloid Interface Sci., 133: 1-21.

15. Loose, M. and Schwille, P. (2009). Biomimetic Membrane Systems to Study Cellular Organization, J. Struct. Biol., 168: 143-151.

16. Gopalakrishnan, G., Rouiller, I., Colman, D.R. and Lennox, R.B. (2009). Supported Bilayers Formed from Different Phospholipids on Spherical Silica Substrates, Langmuir, 25: $5455-5458$.

17. Schonherr, H., Johnson, J.M., Lenz, P., Frank, C.W. and Boxer, S.G. (2004). Vesicle Adsorption and Lipid Bilayer Formation on Glass Studied by Atomic Force Microscopy, Langmuir, 20: 11600-11606.

18. Reimhult, E.; Hook, F.; Kasemo, B. (2003). Intact Vesicle Adsorption and Supported Biomembrane Formation from Vesicles in Solution: Influence of Surface Chemistry, Vesicle Size, Temperature, and Osmotic Pressure, Langmuir, 19: 1681-1691.

19. Richter, R.P., Berat, R. and Brisson, A.R. (2006). Formation of Solid-Supported Lipid Bilayers: An Integrated View, Langmuir, 22: 3497-3505.

20. Haratake, M., Hidaka, S., Ono, M. and Nakayama, M. (2006). An lonic Polymer Beadsupported Lipid System, J. Colloid Interface Sci., 299: 924-927.

21. Haratake, M., Hidaka, S., Ono, M. and Nakayama, M. (2007). Preparation of an Ionexchangeable Polymer Bead Wrapped with Bilayer Membrane Structures for High Performance Liquid Chromatography, Anal. Chim. Acta, 589: 76-83.

22. Kitson, R.E. and Mellon, M.G. (1944). Colorimetric Determination of Phosphorus as Molybdivanadophosphoric Acid, Ind. Eng. Chem. Anal. Ed., 16: 379-383. 
23. Haratake, M. and Sunamoto, J. (2006). Direct Fusion between Poly(ethylene oxide)-lipid Modified Liposomes and Murine Mitotic B16 Melanoma Cells, J. Bioact. Compat. Polym., 21: $503-517$.

24. McAteer, A. and Davis, J.M. in: Davis, J.M. (Ed.) (1994). Basic Cell Culture: A Practical Approach, IRL Press, Oxford, pp.128.

25. Whirtmarsh, A.J. and Davis, R.J. (1998). Structural Organization of MAP-kinase Signaling Modules by Scaffold Proteins in Yeast and Mammals, Trends Biochem. Sci., 23: 481-485. 
Table 1. Particle diameter of liposomes used for the preparation of phospholipid-SA11A complexes.

\begin{tabular}{lcc} 
Phospholipid composition (mol\%) & Particle diameter $^{\mathrm{a}}(\mathrm{nm})$ \\
\hline Phosphatidylserine 100 / Phosphatidylcholine 0 & $44.50 \pm 4.76$ \\
Phosphatidylserine 75 / Phosphatidylcholine 25 & $60.20 \pm 12.79$ \\
Phosphatidylserine 50 / Phosphatidylcholine 50 & $77.75 \pm 12.36$ \\
Phosphatidylserine $\quad 25$ / Phosphatidylcholine 75 & $97.50 \pm 15.93$ \\
Phosphatidylserine $\quad$ 0 / Phosphatidylcholine 100 & $1772.25 \pm 291.36$
\end{tabular}

${ }^{\mathrm{a}}$ Mean \pm standard deviation.

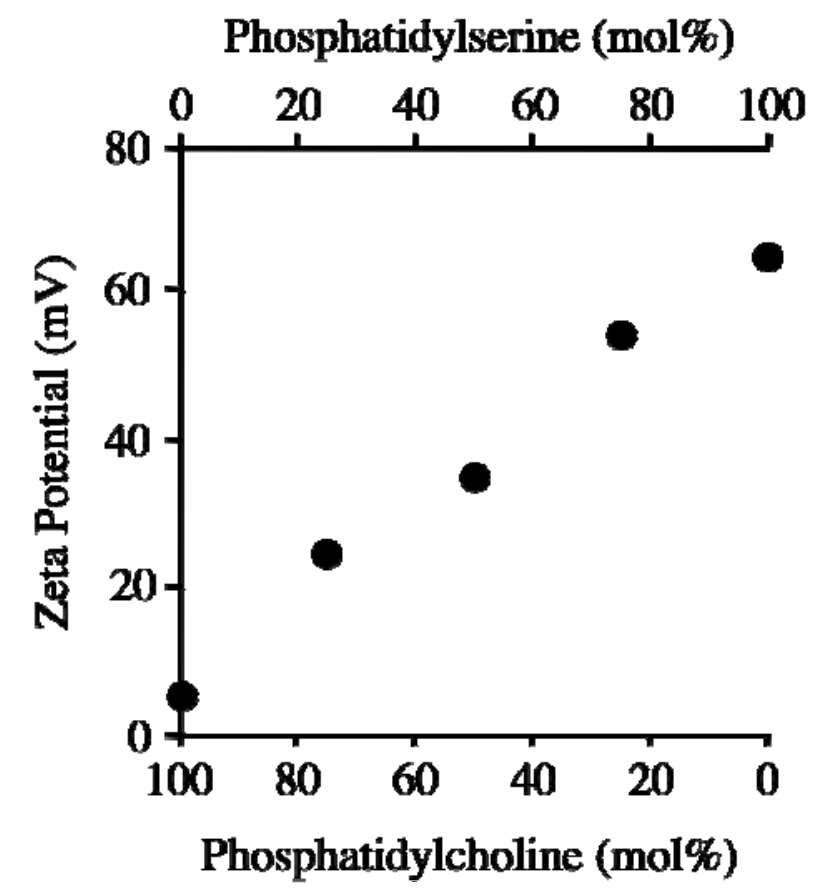

Figure 1. Zeta potential of liposomes used for the preparation of the phospholipidSA11A complexes. 


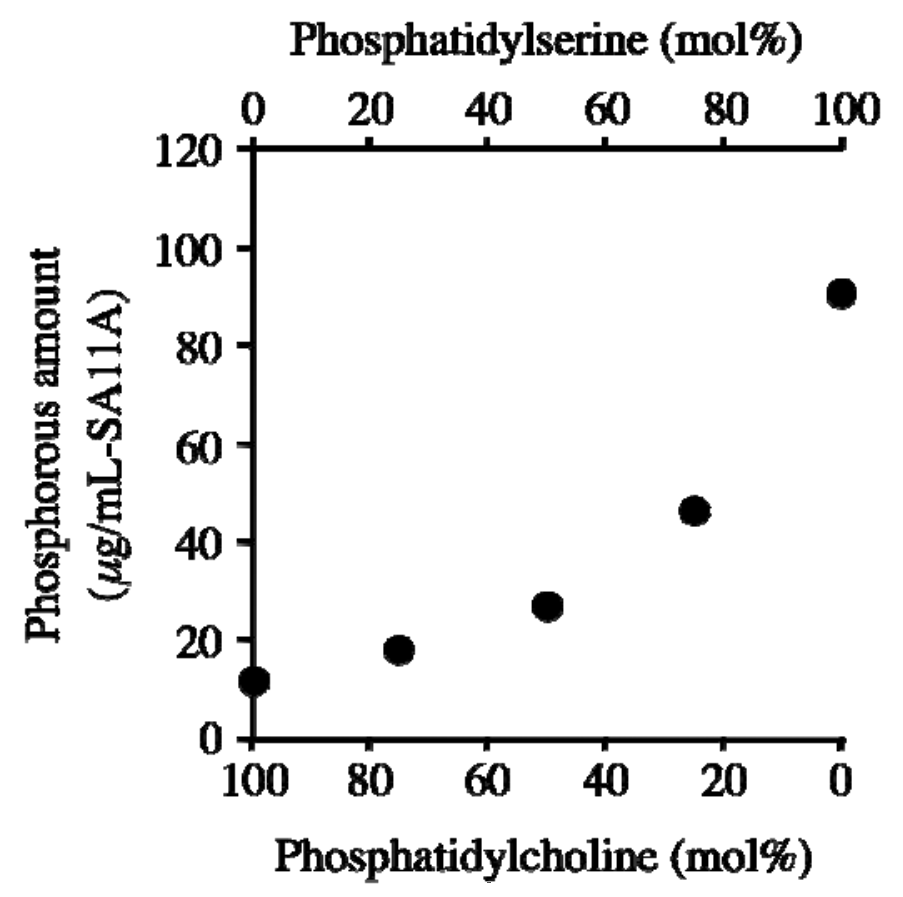

Figure 2. Phosphorous content of the phospholipid-SA11A complexes with varying lipid composition.
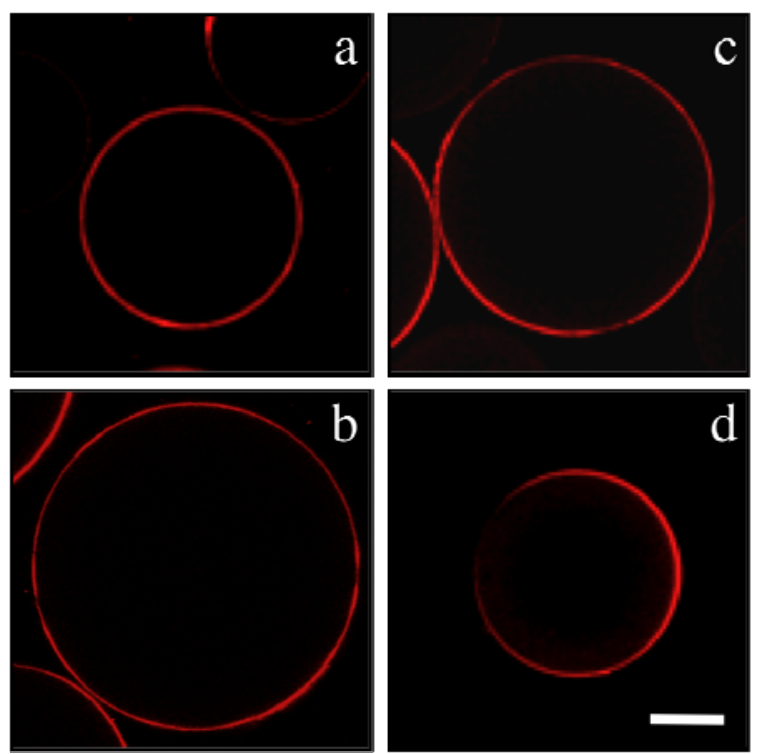

Figure 3. Confocal fluorescence images of octadecylrhodamine-labeled phospholipid-SA11A complexes. Phosphatidylserine (PS) and phosphatidylcholine (PC) composition (PS/PC by mol\%): (a) 20/80, (b) 50/50, (c) 80/20, (d) 100/0, Scale bar in panel $\mathrm{d}$ represents $0.1 \mathrm{~mm}$. 

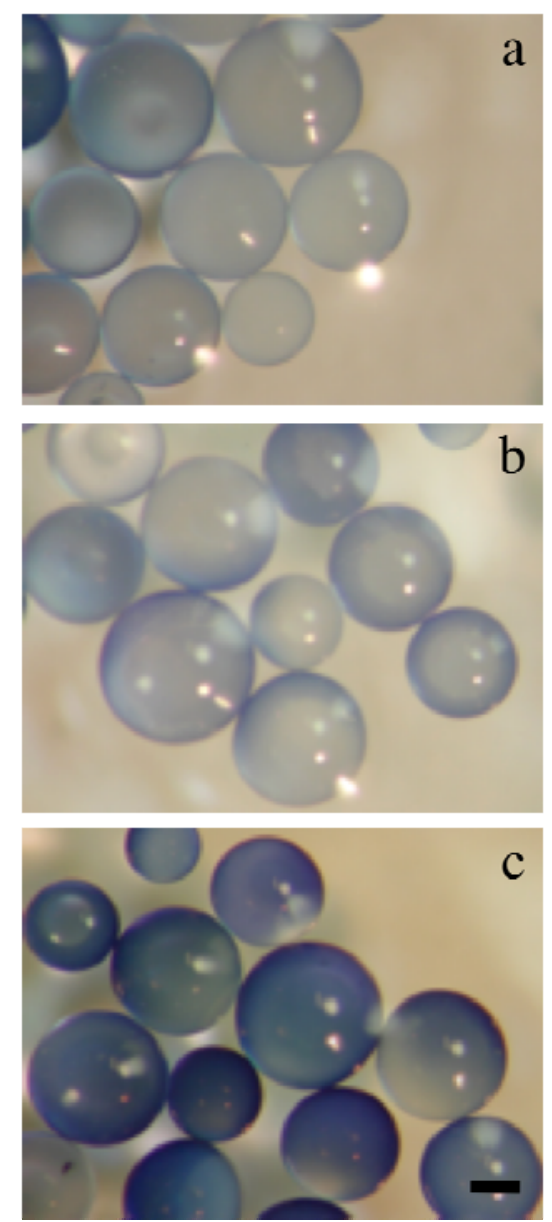

Figure 4. Light micrographs of the phospholipid-bead complexes after treatment with $0.002 \mathrm{w} / \mathrm{v} \%$ trypan blue solution. Phosphatidylserine (PS) and phosphatidylcholine (PC) composition (PS/PC by mol\%): (a) 50/50, (b) 100/0, (c) 0/0 (phospholipid-free SA11A). Objective magnification: $\times 10$, Optical zoom: $\times 4$, Scale bar in panel $c$ represents $0.2 \mathrm{~mm}$. 